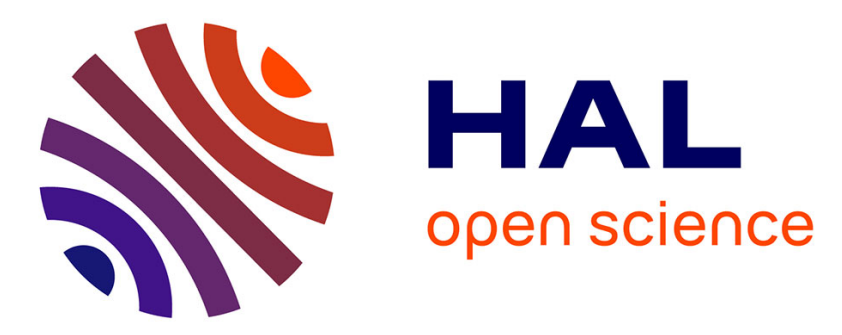

\title{
Le Castellan (Istres, Bouches-du-Rhône) : Résultats de prospection géophysique
}

Ian Armit, Tim Horsley, Frédéric Marty

\section{To cite this version:}

Ian Armit, Tim Horsley, Frédéric Marty. Le Castellan (Istres, Bouches-du-Rhône): Résultats de prospection géophysique. Documents d'archéologie méridionale, 2017, Documents d'Archéologie Méridionale, 39, pp.75-82. 10.4000/dam.5690 . halshs-03406976

\section{HAL Id: halshs-03406976 \\ https://shs.hal.science/halshs-03406976}

Submitted on 28 Oct 2021

HAL is a multi-disciplinary open access archive for the deposit and dissemination of scientific research documents, whether they are published or not. The documents may come from teaching and research institutions in France or abroad, or from public or private research centers.
L'archive ouverte pluridisciplinaire HAL, est destinée au dépôt et à la diffusion de documents scientifiques de niveau recherche, publiés ou non, émanant des établissements d'enseignement et de recherche français ou étrangers, des laboratoires publics ou privés. 
Assumpció TOLEDO I MUR, ANNE LAGARRIGUE

Les ensembles céramiques du Bronze ancien et du Bronze final IIIa d'El Camp del Viver

(Baho, Pyrénées-Orientales)

- Fabrice PONS, Sébastien PANCIN, Roberta BEVILACQUA, Hélène MARTIN

Un habitat de la fin du premier âge du Fer dans le Toulousain: le site de Sauzas à Blagnac (Haute-Garonne)

- IAN ARMIT, TIM HORSLEY, FRÉDÉRIC MARTY.

Le Castellan (Istres, Bouches-du-Rhône) : résultats de prospection géophysique

- AnNie MONTÉCINOS, RÉJAne ROURE, SÉBAstien MUNOS

L’agglomération protohistorique de Mèze (Hérault): nouvelles données sur l'âge du Fer

- Alexandre BeYlier, Anne-Marie CURÉ, Cécile DUbOSSE, Éric GAILlEDRAT . avec la collaboration de SÉBASTIEN MUNOS et de SÉvERINE SANZ-LALIBERTÉ

Le dépôt de la «fouille 47» du Cayla de Mailhac (Aude): un ensemble du V $\mathrm{V}^{\mathrm{e}}$. av. n. è. à caractère rituel?

- Axel CAUVIN

La partie méridionale du premier village du quartier de l'Île à Martigues (Bouches-du-Rhône) entre le milieu du $\mathrm{V}^{\mathrm{e}}$ s. et la fin du $\mathrm{III}^{\mathrm{e}}$ s. av. J.-C.

- LoḮ BUFFAT, Bernard DEDET.

avec la collaboration de CRISTINA GARCIA DALMAU et AlEXANDRE AYASSE

L'ensemble funéraire du second âge du Fer de la Grange Blanche à Courthézon (Vaucluse)

- Patrice Courtaud, Elisabeth ROUSSEAU, Henri DUdAy, Philippe BOISSinOt

Les restes humains de l'âge du Fer de Roquepertuse (13) : fouilles anciennes et récentes 



\section{Le Castellan (Istres, Bouches-du-Rhône) : résultats de prospection géophysique}

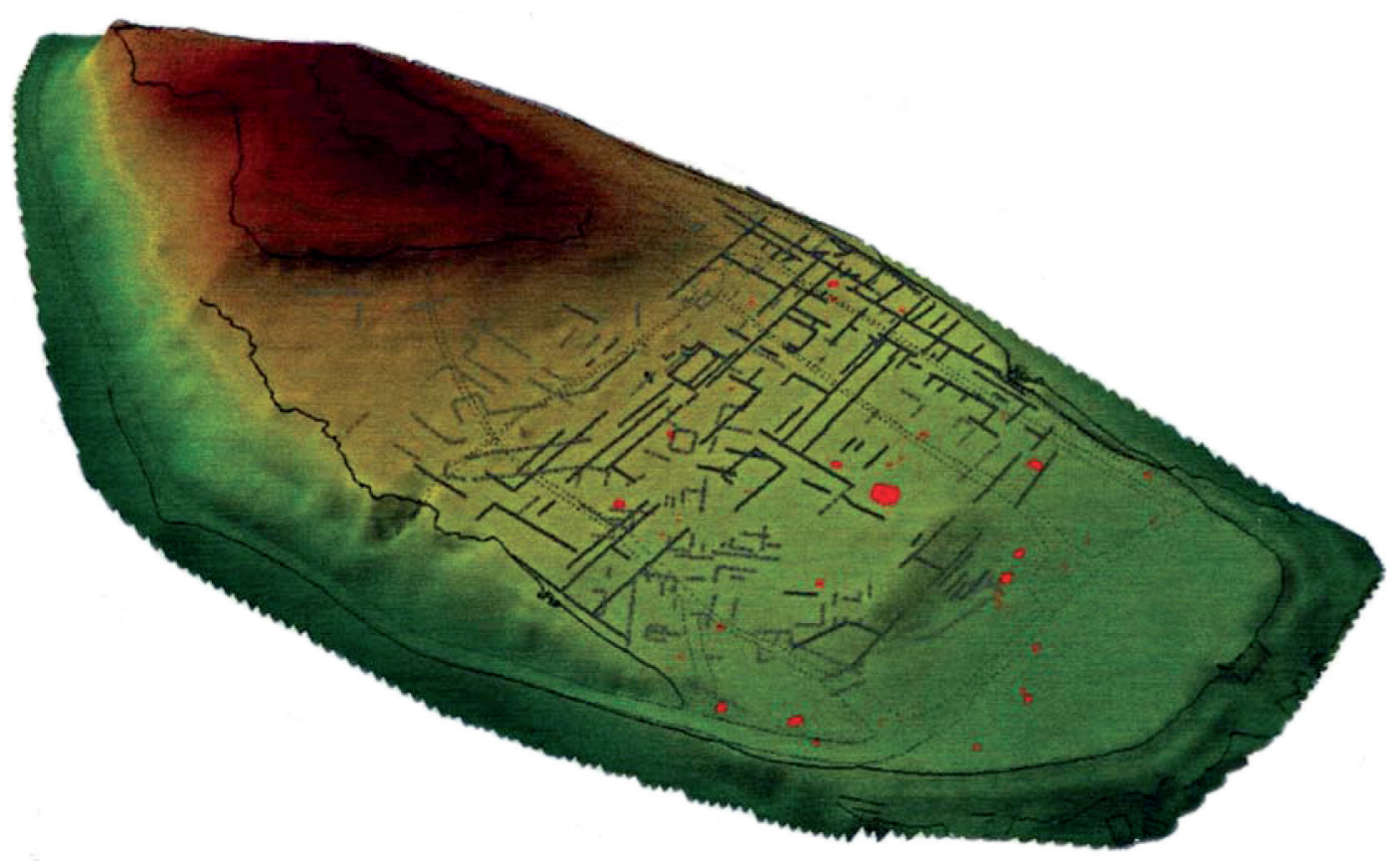

Résumé:

Deux campagnes de prospection géophysique (magnétique, électrique et géoradar) ont été conduites sur l'oppidum gaulois du Castellan (Istres, Bouches-du-Rhône) afin de déterminer la validité de ces méthodes pour les agglomérations de l'âge du Fer dans la région. L'étude a révélé plusieurs anomalies importantes, généralement orientées parallèlement ou perpendiculairement les unes aux autres. Celles-ci correspondent au type de réponse attendu pour des vestiges de murs en pierres et de fondations enfouis. Cette interprétation est confortée par la présence de murs visibles en coupe, du côté ouest du site, montrant la même orientation que les anomalies. Ces données suggèrent l'existence d'une trame urbaine composée d'espaces bâtis et d'espaces viaires. Un bâtiment particulièrement important a été identifié: une anomalie de résistivité élevée, de forme rectiligne et très distincte, se situe au centre du secteur d'étude. Cette anomalie semble indiquer la présence d'un bâtiment en pierre enfoui composé de trois pièces. En conclusion, les résultats se révèlent fort encourageants pour la poursuite des prospections géophysiques dans la région.

\section{Mots clés:}

prospection géophysique, prospection magnétique, prospection électrique, géoradar, oppidum, urbanisme

\section{Abstract:}

Two seasons of geophysical prospection (magnetic, resistance and ground-penetrating radar) were conducted at the Iron Age oppidum of Le Castellan, Istres, Bouches-du-Rhône, in order to determine the utility of these techniques for sites in this region. The survey revealed numerous linear anomalies, many of which are aligned to form a regular grid pattern. These are the sorts of responses one might expect from the remains of buried stone wall foundations; this interpretation is supported by the presence, on the west side of the site, of exposed walls on the same alignment as certain of the geophysical anomalies. Overall, the evidence suggests a network of buried buildings and road-ways across the oppidum. One particularly substantial building was identified towards the centre of the site, through the presence of a strong high resistance anomaly of distinctly rectilinear form. It appears to represent the remains of a buried stone building with three rooms. In conclusion, the results provide strong encouragement for the further application of geophysical survey in this region.

Keywords:

geophysical prospection, magnetic prospection, resistance prospection, ground-penetrating radar, oppidum, urbanism 


\section{Le site}

L'oppidum du Castellan est implanté sur un promontoire rocheux - dont le substrat est composé d'une molasse miocène - s'avançant dans la partie méridionale de l'étang de l'Olivier, au nord du centre ancien d'Istres. Ce promontoire est formé d'un plateau sommital étroit à la cote $39 \mathrm{~m}$, culminant à 40,85 m NGF. En contrebas, du côté nord-est, se situe un replat d'environ 10 à $15 \mathrm{~m}$ de largeur, à une altitude approximative de 32-35 m. Le reste du site, au nord, peut être divisé en deux terrasses en pente douce, séparées par un faible talus recouvert de pins et d'oliviers.

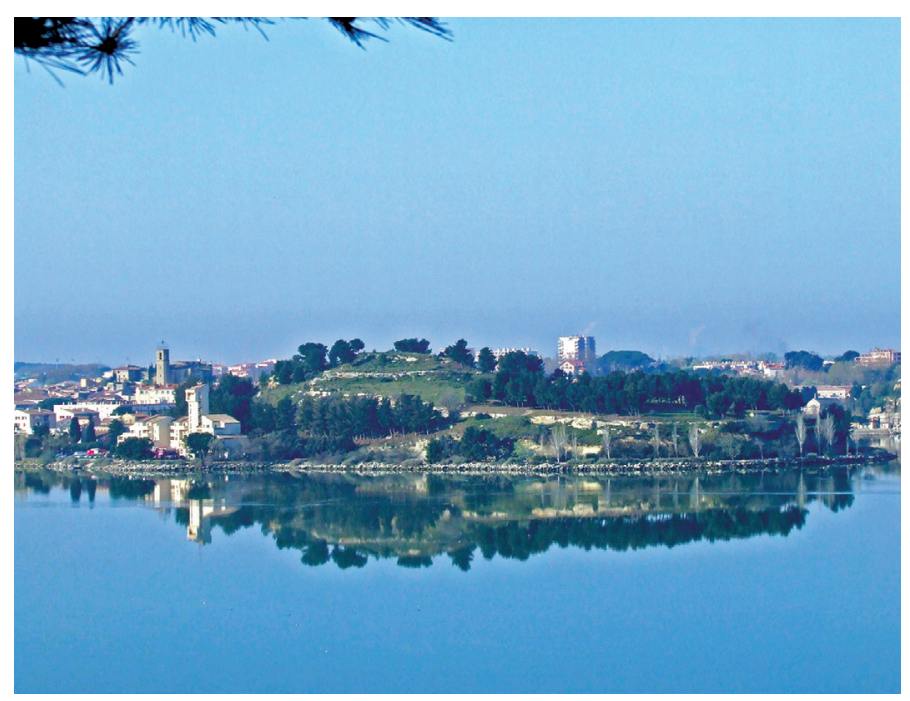

I 1 La colline du Castellan, à Istres : vue vers le sud-ouest.

L'habitat de hauteur (fig. 1-2) a été fondé au début du $\mathrm{VI}^{\mathrm{e}} \mathrm{s}$. av. J.-C. Il a connu une occupation dense aux $\mathrm{VI}^{\mathrm{e}}-\mathrm{V}^{\mathrm{e}} \mathrm{s}$ et aux $\mathrm{II}^{\mathrm{e}} \mathrm{I}^{\mathrm{er}} \mathrm{S}$. av. J.-C., ponctuée par une période de relative latence aux $\mathrm{IV}^{\mathrm{e}}-\mathrm{III}^{\mathrm{e}} \mathrm{s}$. av. J.-C. (Marty 2002). Après un abandon de quelques décennies, il est de nouveau réoccupé aux I ${ }^{\mathrm{er}}$ et $\mathrm{II}^{\mathrm{e}} \mathrm{s}$. ap. J.-C., époque à laquelle se développe, sur son piémont ouest, une agglomération rurale gallo-romaine (Marty, Chevaux 2017). Les fouilles anciennes conduites au sommet ont révélé des murs de bâtiments en pierre et ont livré une riche collection de mobiliers protohistoriques, malheureusement privés de stratigraphie. L'emplacement précis de la plupart de ces bâtiments est perdu. Plus récemment, les fouilles scientifiques menées en 2000 , ont mis au jour, à l'extrémité nord du plateau, une habitation détruite à la fin du II ${ }^{\mathrm{e}} \mathrm{s}$. av. J.-C. et un rempart-terrasse de la première moitié du Irr s. av. J.-C. Ces recherches ont permis d'obtenir une première stratigraphie sur le gisement. Toutefois, l'organisation de l'agglomération protohistorique demeurait entièrement inconnue.

Rares sont les vestiges bâtis actuellement discernables à la surface du sol. Cependant, deux sections courtes de murs, découvertes en 1976, restent encore visibles sur le côté occidental du site (fig. 3). Elles forment l'angle d'une construction enfouie entre $1 \mathrm{~m}$ et $1,40 \mathrm{~m}$ de la surface du sol actuel, sous une couche de remblai de $40 \mathrm{~cm}$ d'épaisseur, contenant des débris de coquilles d'huîtres fossiles. Du côté oriental du promontoire, non loin du sommet, une autre section courte de mur est visible. D'autres murs ont, semble-t-il, également été découverts lors des fouilles anciennes du milieu du $\mathrm{XX}^{\mathrm{e}} \mathrm{s}$. (sondages alignés dans le sens est / ouest le long de la cote des $25 \mathrm{~m}$, sur toute la largeur du plateau), mais n'ont jamais fait l'objet de relevés.

\section{La prospection géophysique}

En 2007 et 2008, deux campagnes de prospection géophysique - électrique (résistivité), magnétique et géoradar - ont été conduites sur la colline ${ }^{1}$. Leur implantation est donnée dans la figure 4. Un relevé topographique utilisant un SIG a été mené conjointement à la prospection géophysique. Les visualisations en 3D sont présentées dans la figure 5 avec les résultats de résistivité.

Pour la prospection électrique, une configuration twinprobe a été utilisée avec une distance de mobile probe de $50 \mathrm{~cm}$ et un espacement des remote electrodes d'environ 2 $\mathrm{m}$. Cet espacement de $50 \mathrm{~cm}$ correspond à une profondeur d'investigation comprise entre $75 \mathrm{~cm}$ et $1 \mathrm{~m}$ s'accordant très probablement avec la localisation de la majorité des structures archéologiques. Les données ont été recueillies à $50 \mathrm{~cm}$ d'intervalle le long de lignes parallèles espacées de $1 \mathrm{~m}$ ou de $50 \mathrm{~cm}$. Une résolution élevée a été employée à titre expérimental sur un secteur de 40 x $25 \mathrm{~m}$ afin de vérifier les avantages de la méthode avec un intervalle plus rapproché. Les données de résistivité ont été automatiquement enregistrées au moment de l'insertion des électrodes dans le sol à une vitesse d'enregistrement moyenne. Les lignes ont été prospectées les unes après les autres en suivant des directions alternes, c'est-à-dire selon la méthode dite «en zigzag».

Pour la prospection magnétique, un gradiomètre fluxgate FM36 de Geoscan Research a été utilisé sur une surface de 0,9 ha. Les données ont été recueillies à $12,5 \mathrm{~cm}$ d'intervalle le long de lignes parallèles, espacées de $50 \mathrm{~cm}$, et ont 


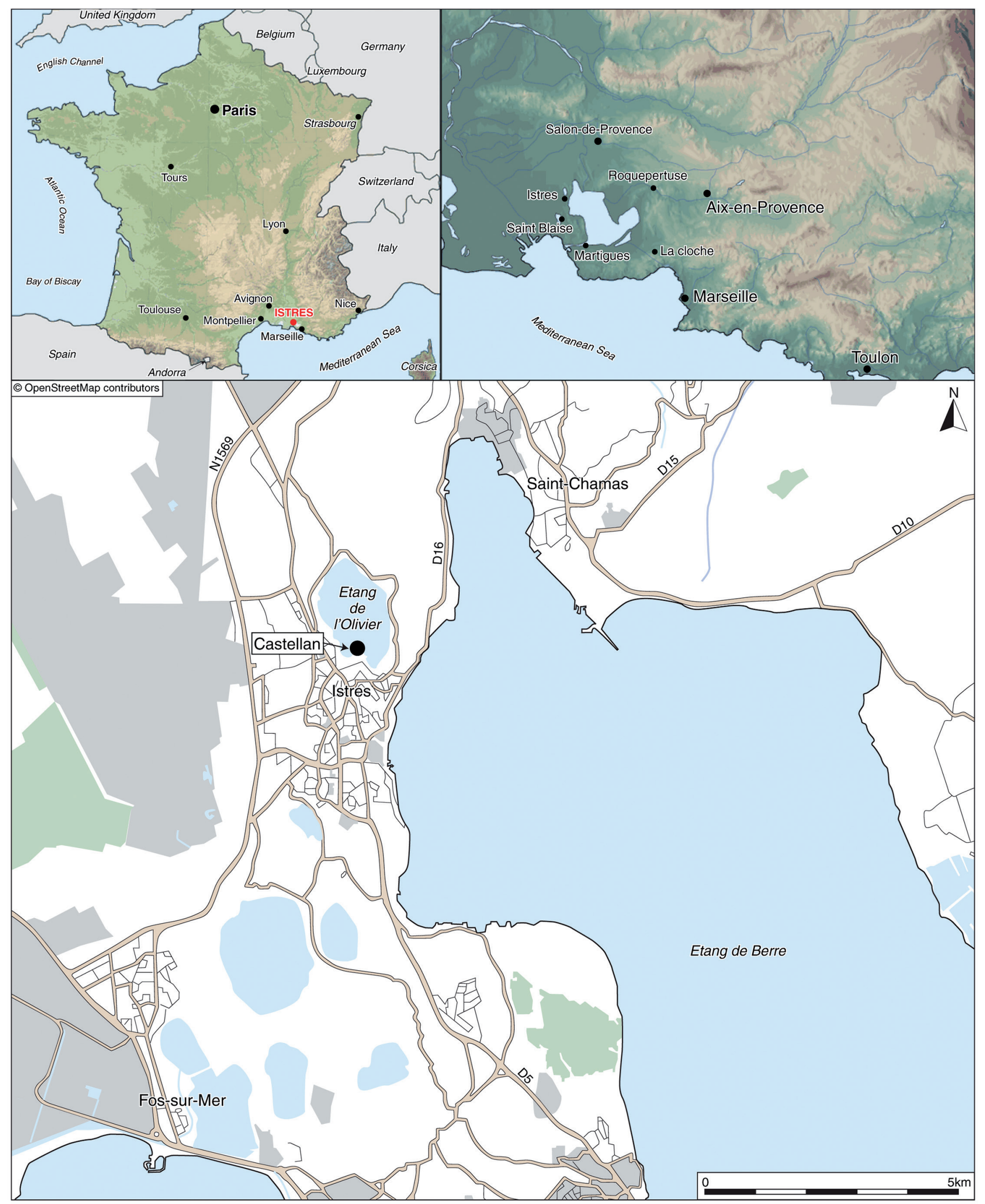

I 2 Localisation du site. 


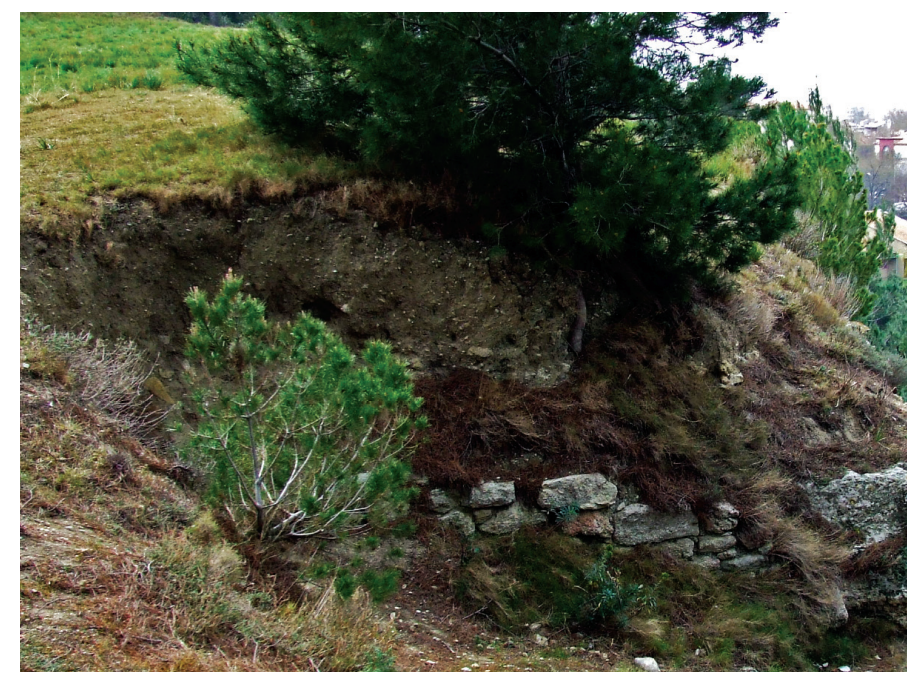

I 3 Murs découverts en 1976 sur le côté occidental du plateau : vue vers le sud.

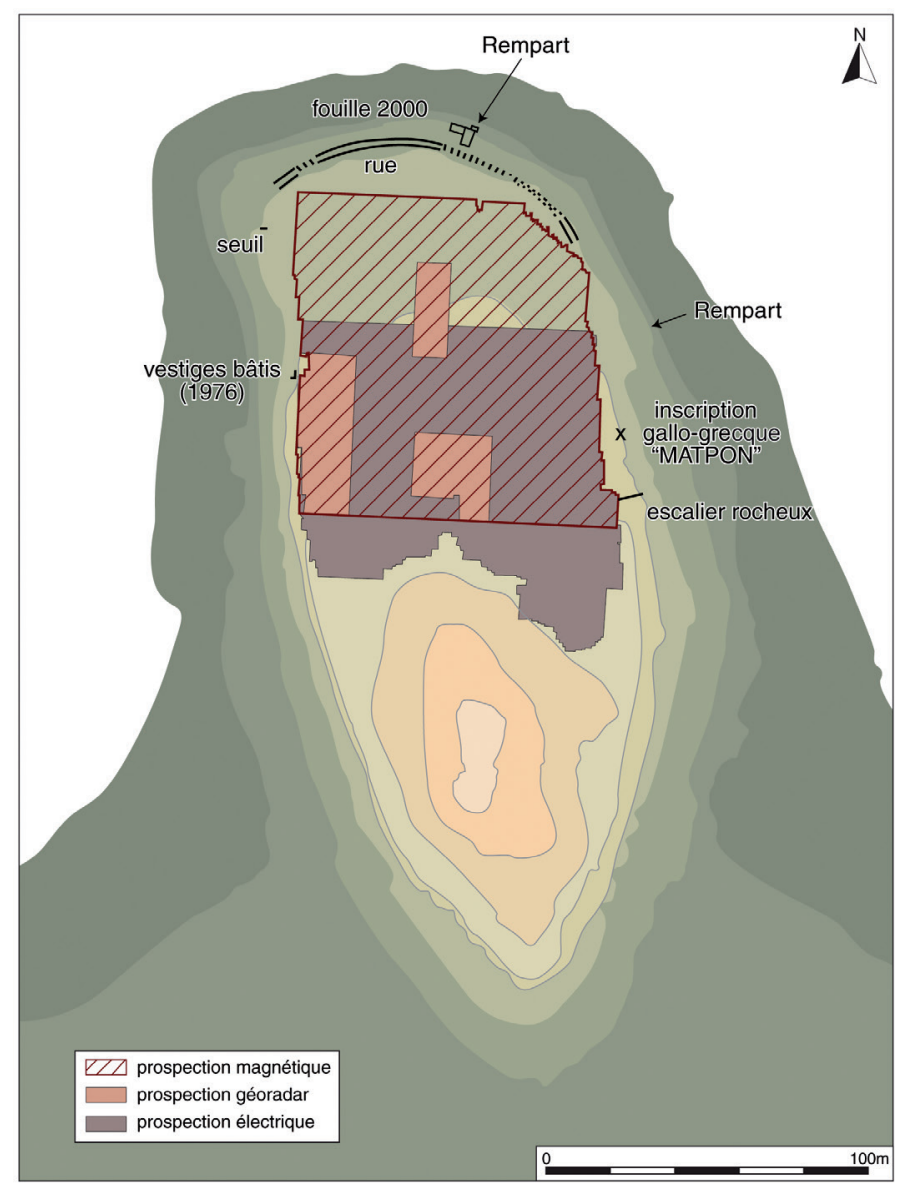

4 Le Castellan : zones de prospection.

été enregistrées en marchant tout en utilisant une méthode automatisée. Toutes les lignes ont été parcourues dans la même direction, en mode «parallèle». Après l'achèvement de chaque aire de prospection, l'organisation électronique et mécanique de l'instrument a été ajustée pour rectifier

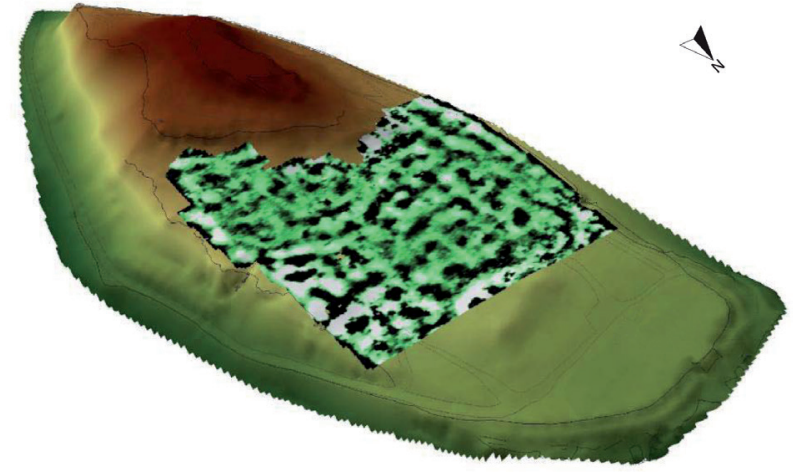

I 5 Visualisation en 3D (par Robert Friel) avec les résultats de résistivité.

la dérive. Le magnétomètre a été réglé à une sensibilité d'enregistrement de 0,1 nT. Des informations supplémentaires sur cette technique sont rassemblées dans Aspinall et al. 2008; Gaffney, Gater 2003 et Clark 1996.

Toutes les données ont été traitées en utilisant Geoplot 3.00. Des détails supplémentaires sont consignés dans nos rapports inédits (Armit et al. 2007; 2008). Ces deux méthodes ont fait leurs preuves tant pour la recherche archéologique de vestiges bâtis enfouis que de puits et de fossés. La complémentarité des résultats acquis facilite l'interprétation des anomalies géophysiques. Les résultats de la prospection géoradar, non décrits ici, ont permis de vérifier la validité de ces deux méthodes.

\section{Résultats}

\subsection{Prospection électrique}

Une gamme de réponses d'intensités différentes a été détectée, dont plusieurs forment des alignements réguliers (fig. 6). Le résultat correspond exactement à ce que pourraient produire des vestiges de murs en pierre enfouis. L'hypothèse d'une origine géologique ne doit pas pour autant être exclue dans le cas de certaines anomalies: le substrat molassique peut en effet produire des anomalies de résistivité régulières en raison d'un drainage différentiel du sous-sol induit par la présence de fissures entre les dalles solides du rocher. Il n'est pas toujours possible de distinguer anomalies naturelles et anomalies d'origine anthropique. Cependant, nombre d'entre elles ont certainement une origine archéologique. Parmi celles-ci, l'anomalie (fig. 6d, élément 2) est certainement celle dont l'origine archéologique est la plus 


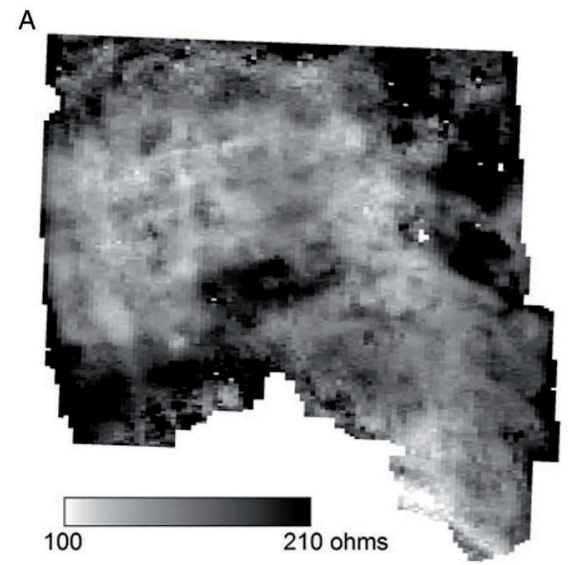

$\Lambda$

B
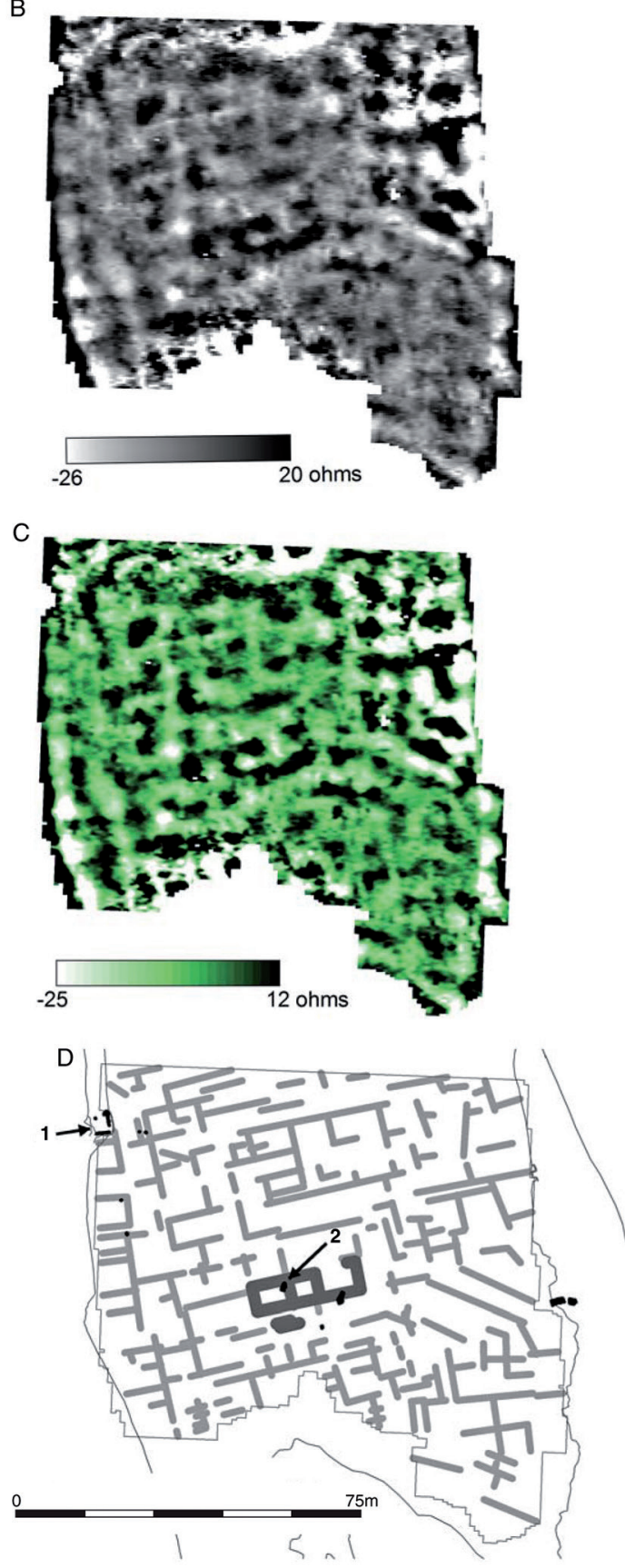

I 6 Résultats de la prospection électrique : a. données électriques brutes après 'despiking' (valeurs de 100 (blanc) à 210 ohms (noir)); b. données après l'application d'un filtre 'High Pass' et interpolation (valeurs de -26 (blanc) à 20 ohms (noir)); c. données après l'application de deux filtres 'High Pass' identiques et interpolation: (valeurs de -24 (blanc) à 18 ohms (noir)); d. interprétation des résultats.

probable. Sa forme et ses dimensions suggèrent l'existence d'un bâtiment rectangulaire comportant trois pièces, dont une centrale mesurant environ 10 × $6 \mathrm{~m}$. Il est intéressant de noter qu'un fragment de seuil en molasse gît à la surface du sol, au-dessus du mur ouest de la pièce centrale (fig. 7). Plusieurs anomalies linéaires situées à l'ouest du site possèdent une orientation similaire.

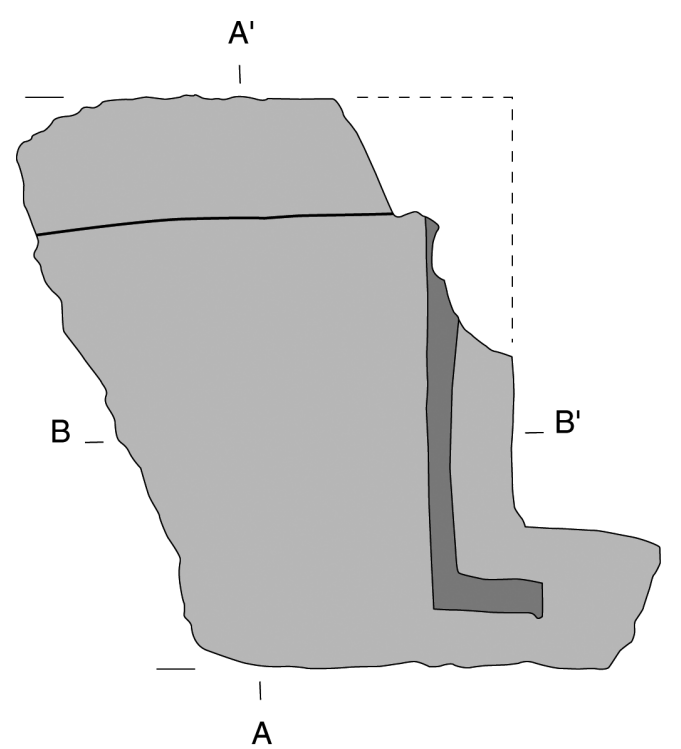

A
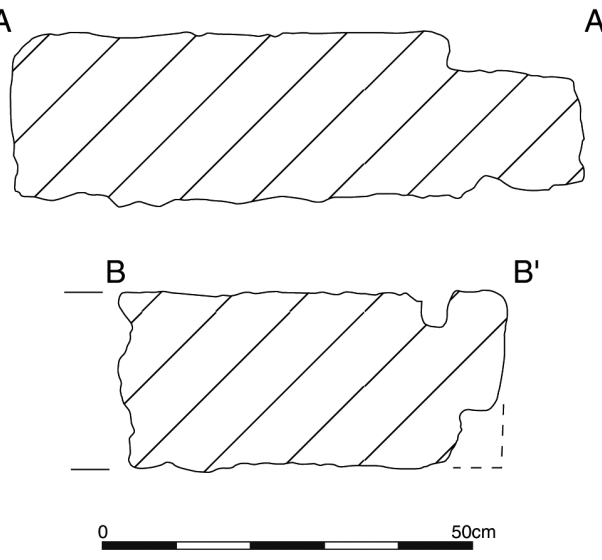

7 Fragment de seuil en pierre (relevé et DAO F. Marty). 


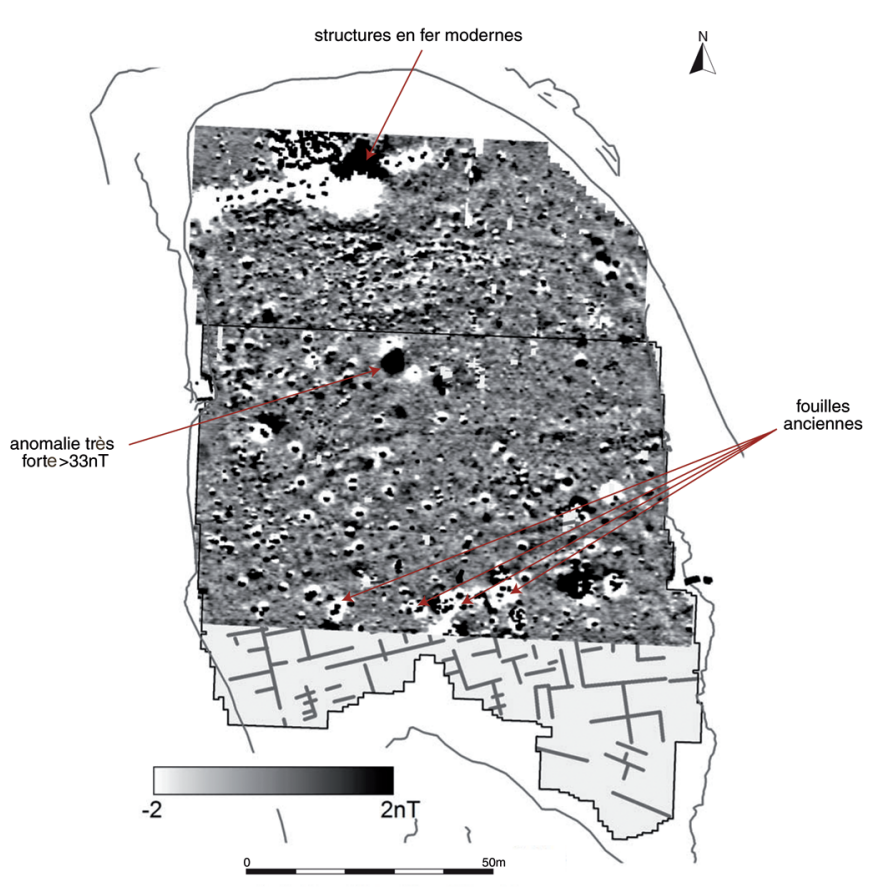

I 8 Résultats de la prospection magnétique.

Un filtre High-Pass a été utilisé afin de percevoir les anomalies faiblement positives (fig. 6c). L'image obtenue rappelle fortement l'organisation des rues et îlots d'oppida proches tels qu'Entremont (Arcelin 2006; Armit et al. 2012) ou La Cloche (Chabot 2004).

\subsection{Prospection magnétique}

Des réponses magnétiques, distinctes et nombreuses, ont été détectées (fig. 8). Ces anomalies bipolaires intenses sont généralement supérieures à $\pm 20 \mathrm{nT}$ et correspondent le plus souvent à des débris ferreux modernes. Bien qu'il soit possible que certaines de ces réponses soient causées par des objets en fer anciens, on ne peut les distinguer de celles dues aux objets récents. Au nord du secteur, certaines anomalies très claires correspondent à des structures en fer modernes : bancs, restes d'une clôture et béton armé.

Malgré ces difficultés, il est possible de distinguer, sur la plus grande partie du secteur prospecté, des traces linéaires fines parallèles et perpendiculaires les unes aux autres. Les réponses linéaires, négatives et faibles ( 0 et $-1.5 \mathrm{nT})$, correspondent probablement à des murs enfouis (Gaffney et al. 2000; Horsley 2007). Celles-ci se produisent lorsque les matériaux de construction sont moins magnétiques que

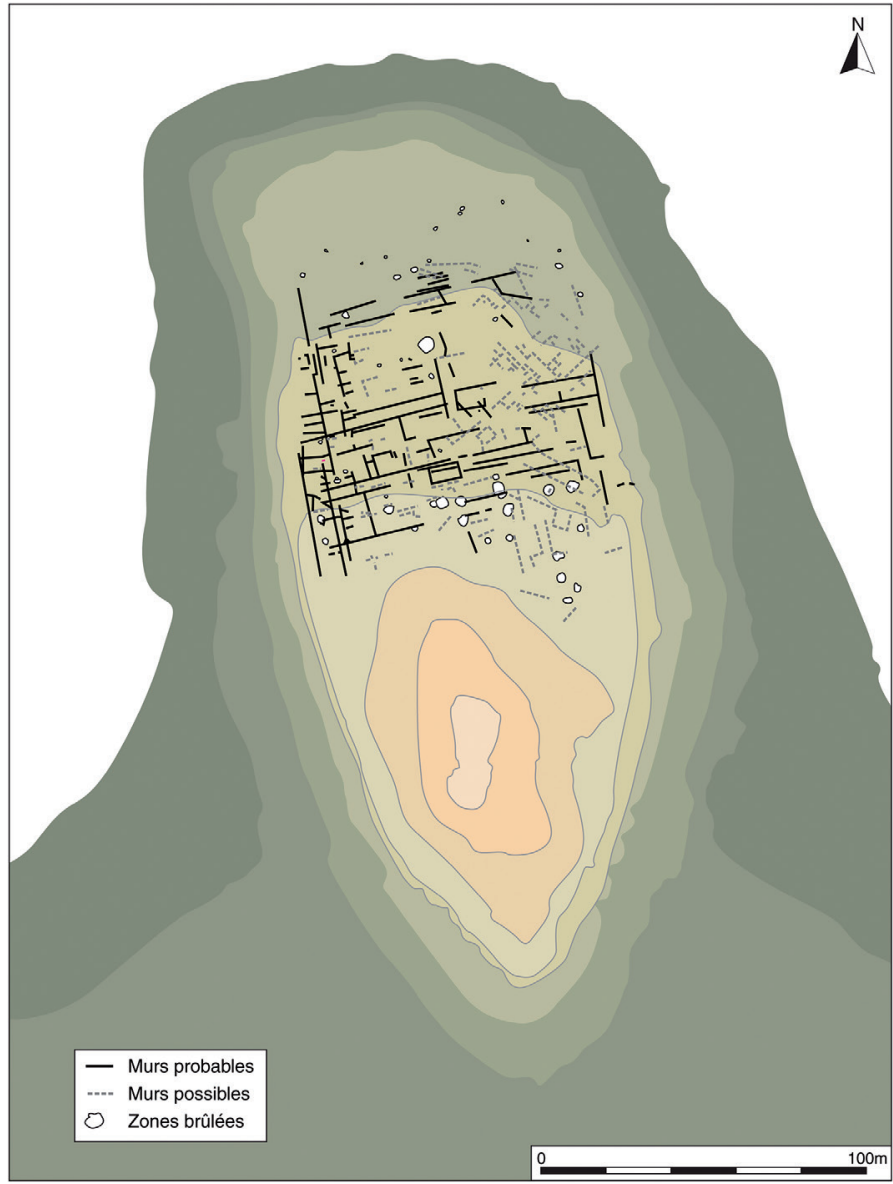

I 9 Interprétation globale de la prospection géophysique.

le sol environnant. Au Castellan, ces résultats indiquent que la pierre utilisée pour construire les murs (probablement la molasse locale) a une valeur magnétique basse comparée au sol environnant. Ce contraste magnétique est peu élevé. Deux orientations distinctes peuvent être identifiées: le sud et l'ouest de l'habitat sont principalement

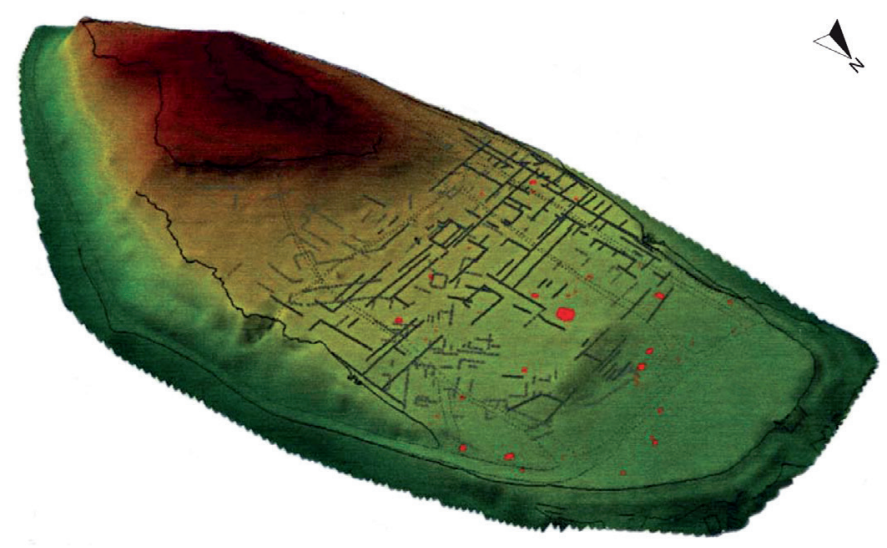

I 10 Interprétation globale de la prospection géophysique superposée au modèle topographique. 
caractérisés par des alignements de tendance ouest-sudouest / est-nord-est. Les réponses linéaires au nord-est ont une orientation nord-ouest / sud-est. Il est intéressant de noter que ce relevé est comparable aux alignements observés lors de la prospection électrique. En effet, lorsque l'on compare les deux séries de données, on observe une forte corrélation des résultats.

En certains endroits, il apparaît évident que le magnétisme du sol est induit par l'occupation humaine. Plusieurs anomalies positives discrètes, supérieures à $5 \mathrm{nT}$, sont visibles sur l'ensemble des données. Alors que certaines de ces réponses peuvent être la conséquence d'objets en fer enfouis, la majorité est interprétée comme l'indice d'emplacements de foyers. Quelques anomalies positives plus étendues et plus intenses ont également été détectées. La plus forte de ces mesures, d'environ $5 \mathrm{~m}$ de diam., est supérieure à $33 \mathrm{nT}$. Une telle réponse suggère l'existence d'une zone rubéfiée importante signalant la trace d'une activité artisanale (un four, par exemple), ou bien la présence d'une construction à architecture de terre incendiée.

\section{Conclusion}

La prospection géophysique a permis de détecter une série d'anomalies sur le site du Castellan (fig. 9-10). Les résultats de la prospection électrique montrent, notamment, une répartition régulière d'anomalies linéaires orthogonales sur l'ensemble de l'habitat. Alors que certaines d'entre elles peuvent être d'origine naturelle, la plupart correspondent probablement à des vestiges de murs en pierre enfouis. Ces données suggèrent l'existence d'une trame urbaine composée d'espaces bâtis et d'espaces viaires, sur la colline.
Un bâtiment particulièrement important a été identifié. L'intensité du signal indique peut-être qu'il se trouve plus près de la surface du sol actuel, à moins qu'il ne s'agisse d'une construction plus conséquente (bâtiment public?).

La prospection magnétique a aussi révélé une trame régulière d'anomalies linéaires que l'on peut interpréter de manière fiable comme l'indication de murs en pierre et de fondations enfouis. En raison du faible degré des réponses et de l'abondance des débris de fer modernes, il n'a pas été possible de tracer chaque mur. Cependant, les résultats confortent amplement nos interprétations précédentes. Ils indiquent, en outre, la présence d'alignements de murs orientés différemment dans certaines zones, permettant de suggérer l'existence de plusieurs phases de construction (premier puis second âges du Fer?).

Enfin, l'existence d'une éventuelle enceinte séparant les deux terrasses principales peut à présent être écartée. Les rares éléments de rempart ayant échappé à l'érosion et aux carrières de sable sont situés en limite de plateau, au nord et au nord-est, en dehors des zones prospectées.

IAN ARMIT

Archaeological Sciences, University of Bradford, Richmond Rd, Bradford BD7 1DP, United Kingdom

TIM HORSLEY

Department of Anthropology, Northern Illinois University, DeKalb, IL 60115, United States

FRÉDÉRIC MARTY

Métropole Aix-Marseille Provence / Territoire Istres Ouest Provence / Pôle Intercommunal du Patrimoine Culturel, chemin du Rouquier, BP 10647, 13808 Istres cedex, France

1. La prospection a été subventionnée par la British Academy. Les auteurs remercient Robert Friel pour la création des images topographiques ainsi que le Dr Chris Gaffney, le Dr Andrew Parkyn et Dr Fiona Shapland pour leur aide. 
Arcelin 2006: ARCELIN (P.) - Avant Aquae Sextiae: l'oppidum d'Entremont In : MOCCI (F.), NIN (N.) - Aix-en-Provence, Pays d'Aix, Val de Durance (Carte Archéologique de la Gaule $N^{\circ}$. 13/4). Paris, Fondation Maison des Sciences de l'Homme, 2006, p. 125-68

Armit et al. 2007: ARMIT (I.), HORSLEY (T.), MARTY (F.) - Le Castellan, Istres, Bouche-du-Rhône: Résultats de prospection géophysique, 2-6 avril, 2007. Rapport au SRA, inédit, 2007.

Armit et al. 2008: ARMIT (I.), HORSLEY (T.), MARTY (F.) - Le Castellan, Istres, Bouche-du-Rhône: Résultats de prospection géophysique, 18-27 mars, 2008. Rapport au SRA, inédit, 2008.

Armit et al. 2012: ARMIT (I.), GAFFNEY (C.), HAYES (A.) - Space and movement in an Iron Age oppidum: integrating geophysical and topographic survey at Entremont, Provence, Antiquity 86, 331, p. 191-206.

Aspinall et al. 2008: ASPINALL (A.), GAFFNEY (C.), SCHMIDT (A.) - Magnetometry for Archaeologists. Walnut Creek, AltaMira Press, 2008.

Chabot 2004: CHABOT (L.) - L'Oppidum de la Cloche: Les Pennes-Mirabeau, Bouches-du-Rhône. Montagnac, Mergoil, 2004 (Protohistoire Européenne 7).
Clark 1996: CLARK (A. J.) - Seeing Beneath the Soil. London, Batsford, 1996.

Gaffney, Gater 2003: GAFFNEY (C. F.), GATER (J. A.) - Revealing the Buried Past. Geophysics for Archaeologists. Stroud, Tempus Publishing Ltd., 2003.

Gaffney et al. 2000: GAFFNEY (C. F.), GATER (J. A.), LINFORD (P.), GAFFNEY (V. L), WHITE (R.) - Large-scale systematic fluxgate gradiometry at the Roman city of Wroxeter, Archaeological Prospection, 7, 2, 2000, p. 81-99.

Horsley 2007: HORSLEY (T. J.) - Malton Roman Fort, Malton North Yorkshire. Report on Geophysical Surveys, July-August 2007. University of Bradford, inédit, 2007.

Marty 2002: MARTY (F.) - L'habitat de hauteur du Castellan (Istres, B.-duRh.) à l'âge du Fer. Étude des collections anciennes et recherches récentes, Documents d'Archéologie Méridionale, 25, 2002, p. 129-169.

Marty, Chevaux 2017 : MARTY (F.), CHEVAUX (B.) dir. - Une agglomération rurale gallo-romaine des rives de l'étang de Berre : Le Castellan (Istres, Bouches-du-Rhône), (Bibliothèque d'Archéologie Méditerranéenne et Africaine, 23), Éditions Errance, Arles, Centre Camille Jullian, Aix-enProvence, 2017, $299 \mathrm{p}$. 